\title{
Consenso de cuidados respiratorios en enfermedades neuromusculares en niños. Resumen ejecutivo
}

\author{
Consensus statement on respiratory care in pediatric neuromuscular diseases. \\ Executive summary
}

Comité de Neumonología, Grupo de Trabajo de Kinesiología, Grupo de Trabajo de Discapacidad, Comité de Gastroenterología, Comité de Nutrición.

Coordinadora: Dra. Verónica Aguerre

http:/ /dx.doi.org/10.5546/aap.2014.476

Véase el texto completo en formato electrónico.

Las enfermedades neuromusculares (ENM) se caracterizan por la afectación de algún componente de la unidad motora y su principal manifestación es la debilidad muscular. La posibilidad de compromiso del sistema respiratorio varía ampliamente entre las diferentes condiciones y en general aparece en niños con mayor debilidad. La insuficiencia respiratoria aguda asociada a infecciones respiratorias es motivo frecuente de hospitalización y la insuficiencia respiratoria crónica (IRC) es la causa más frecuente de muerte.

En las últimas décadas, se presenció un desarrollo en distintas disciplinas, con un gran avance en la identificación, diagnóstico y tratamiento de las ENM. Se generó una nueva organización de la atención médica, orientada a la atención integral y multidisciplinaria, la prevención de complicaciones y planificación de intervenciones, con un impacto considerable en la calidad y expectativa de vida de los pacientes.

Los contenidos desarrollados en el consenso incluyen:

1. Fisiopatología del compromiso respiratorio en la ENM: La afectación del sistema respiratorio es multifactorialy tiene estrecha relación con el funcionamiento de los músculos respiratorios. Se describen los mecanismos de desarrollo del compromiso respiratorio y las consecuencias de la afectación de cada grupo muscular, así como otros factores intervinientes en la morbilidad respiratoria, como el compromiso esquelético y bulbar.

- Trastornos respiratorios durante el sueño (TRS): En pacientes con ENM la disminución de la eficiencia respiratoria durante el sueño está acentuada por el compromiso de los músculos respiratorios o por afectación de los centros

Correspondencia:

Dr. Fernando Javier Vila: fjvila@hotmail.com

Conflicto de intereses: Ninguno que declarar.

Recibido: 3-7-2014

Aceptado: 30-7-2014 respiratorios. 40 a 70\% de los niños con ENM presentan algún tipo de TRS. Diferentes ENM pueden tener mayor tendencia a desarrollar una u otra forma. El compromiso ventilatorio durante el sueño es el primer paso de todos los pacientes que desarrollan IRC.

- Características del compromiso respiratorio: La gravedad y temporalidad de la afectación de los músculos respiratorios y el riesgo de desarrollar IRC, dependen del diagnóstico. Aun dentro de las patologías que evolucionan a la IRC, la misma puede aparecer en los primeros meses de vida (atrofia muscular espinal, AME tipo I) o tardíamente en la evolución (distrofia muscular de Duchenne, DMD, AME tipo III). Independientemente del momento de inicio, los eventos que sucederán van a seguir un mismo orden cronológico hacia la IRC, variando la temporalidad de los sucesos.

- Problemas extra-pulmonares con impacto respiratorio:

- Alteraciones de la deglución y reflujo gastroesofágico (RGE): Las alteraciones de la deglución aumentan el riesgo de aspiración pulmonar, generan déficit nutricional y morbimortalidad. La disfunción deglutoria tiende a aumentar a medida que progresa la debilidad muscular. Con más frecuencia se traducen en un síndrome aspirativo crónico.

- Nutrición: Los problemas nutricionales asociados a ENM impactan sobre el estado general y el sistema respiratorio. Existe riesgo de malnutrición por dificultades en la alimentación, disfagia, RGE, alto gasto metabólico; o exceso de peso, por la movilidad reducida y tratamiento esteroideo.

- Patología espinal: La escoliosis es común a muchas ENM. Su incidencia refleja generalmente la gravedad de la debilidad asociada. En la AME y la DMD la incidencia es muy alta ( $100 \%$ y $70 \%$, respectivamente); es variable la edad de aparición según la patología. Genera una afectación mecánica en el sistema respiratorio. 
- Compromiso cardíaco: Ocurre en asociación con muchas de las ENM en pediatría. El tipo y extensión de las manifestaciones son específicos del tipo de desorden neuromuscular. La hipoxia crónica puede dar lugar a hipertensión pulmonar.

2. Evaluación y diagnóstico de compromiso respiratorio: Debe realizarse una consulta al neumonólogo pediatra anualmente desde el diagnóstico de la enfermedad. A partir de la detección de compromiso respiratorio estos controles se harán más frecuentes.

Anamnesis y examen físico: Orientados a pesquisar signos y síntomas de compromiso respiratorio, de progresión de la debilidad y mayor afectación de la función respiratoria, de la deglución y de la dinámica del sueño.

Exámenes complementarios:

- Radiografía de tórax de frente

- Pruebas de función pulmonar (PFP): El control funcional es anual, mientras la función pulmonar sea normal. Luego, según el grado de deterioro y riesgo del paciente, se realizarán semestral o trimestralmente. Se recomienda la estimación de la talla mediante la determinación de la envergadura o de la longitud cubital. Incluyen espirometría, medición de función de músculos respiratorios, pico flujo tosido, medición de intercambio gaseoso en vigilia. Se presentan puntos de corte de función pulmonar asociados al desarrollo de complicaciones respiratorias.

- Evaluación en sueño: En todas las evaluaciones clínicas se pesquisarán síntomas de TRS y junto con el examen funcional respiratorio se determinará la necesidad de un estudio de sueño. La evaluación en sueño podrá realizarse mediante una polisomnografía completa, una poligrafía, una oxicapnografía o una oximetría de pulso.

3. Intervenciones respiratorias:

- Asistencia de la tos y eliminación de secreciones: La debilidad de la tos condiciona deterioro del clearance mucociliar ante infecciones inofensivas del tracto superior o episodios de aspiración, lo que conduce a retención de secreciones, neumonías y/o atelectasias. La base del tratamiento kinésico consiste en: asistencia de la tos, hiperinsuflaciones y eliminación de secreciones. Se describen las técnicas, dispositivos existentes, modalidades de implementación y adecuaciones según la edad del paciente.

- Los niños con AME tienen grave debilidad intercostal y abdominal con relativa preservación del diafragma. Se recomienda la posición de decúbito dorsal plano para estos pacientes. En cambio, los niños con DMD presentan marcada debilidad diafragmática, por lo que se benefician con la posición sentada.

- Tratamiento de la insuficiencia respiratoria crónica (IRC); indicaciones de ventilación no invasiva (VNI): La debilidad progresiva es la causa principal de la insuficiencia respiratoria. Inicialmente en la forma de una hipoventilación nocturna y luego se agrega compromiso diurno. La VNI está indicada cuando exista hipoventilación nocturna asociada a síntomas o hipoventilación diurna. Se discuten asimismo otras indicaciones de VNI en ENM en niños.

4. Diagnóstico y evaluación de compromiso de otros sistemas: Se describen métodos de diagnóstico y evaluación de trastorno deglutorio y síndrome aspirativo, situación nutricional, patología espinal, compromiso cardiovascular y las recomendaciones de tratamiento de los mismos.

5. Exacerbaciones en ENM: Alrededor del 90\% de los episodios de fallo respiratorio agudo en pacientes con ENM ocurre en el transcurso de infecciones banales del tracto respiratorio superior y son la causa más frecuente de hospitalización de urgencia. El monitoreo de la oximetría de pulso, administración precoz de antibiótico, intensificación de las maniobras de asistencia de la tos y uso de VNI para mantener una saturación $>95 \%$, han logrado disminuir la frecuencia de hospitalizaciones. No debe administrase en ningún caso oxigeno suplementario en el hogar para mantener una oxígenación normal, debido al riesgo de hipercapnia. Se detallan estrategias de prevención de infecciones respiratorias, conductas indicadas y tratamiento de las mismas. 6. Abordaje interdisciplinario: La enfermedad neuromuscular es una condición crónica, con múltiples comorbilidades, que puede transcurrir durante su evolución por distintas etapas. Cada una de estas etapas requiere la participación de distintos especialistas para la planificación de estrategias terapéuticas y el establecimiento de las prioridades y necesidades de cada momento. El pediatra como médico de cabecera, debe conocer y coordinar los distintos aspectos en el seguimiento del paciente e integrar las consultas con los distintos especialistas, favoreciendo una fluida comunicación entre el equipo tratante y la familia. 7. Cuidados paliativos. Manejo de síntomas. Acompañamiento en el final de la vida. El objetivo principal de los cuidados paliativos es mejorar o mantener la calidad de vida de los pacientes portadores de enfermedades potencialmente mortales. Los objetivos de los cuidados paliativos se logran mediante el manejo de los síntomas, el control del dolor y el soporte psicosocial y espiritual al paciente y a su familia.

Esperamos que el esfuerzo volcado por un gran número de profesionales en la redacción de este Consenso, sirva de guía para el abordaje integral e interdisciplinario del paciente con enfermedad neuromuscular y pueda redundar en una mejor calidad de atención. 